\title{
Lithium battery parameter identification and SOC estimation based on dual-polarized model
}

\author{
Hui Xia, Changlei Li*, Yusang Xu, and Xuehong Liu \\ China Academy of Information and Communications Technology, Energy and environmental \\ assessment Department, 100089Beijing, China
}

\begin{abstract}
An equivalent circuit model of dual polarization (DP) of lithium battery was established according to the application characteristics of lithium battery under the standby condition of $5 \mathrm{G}$ base station. On the basis of the model, recursive least square method with forgetting factor (RLS) was used to identify the model parameters. Finally, the Unscented Kalman filtering (UKF) was used to estimate the SOC of lithium battery in real time with the identified model parameters. The simulation and experimental results showed that the combined estimation using recursive least square method with forgetting factor (RLS) and UKF could greatly improve the estimation accuracy of lithium battery SOC, reduce the estimation error, and further verify the accuracy and effectiveness of the whole modeling.
\end{abstract}

\section{Introduction}

With the vigorous promotion of $5 \mathrm{G}$ technology in China, energy storage technology under the background of new $5 \mathrm{G}$ infrastructure is becoming more and more important. Due to the wide distribution of $5 \mathrm{G}$ micro base stations, the power system is difficult to meet its requirements, so many base stations have been used energy storage systems to ensure continuous and stable power transmission. By configuring energy storage batteries for the communication base station network, the energy storage batteries can improve the power quality when the power supply system is normally powered, and continue to supply power to the load as a backup power source when the power supply fails to ensure the continuous normal operation of the equipment, thus forming a huge distributed. The energy storage system realizes peak-shaving and valley-filling of base stations and reduces base station construction and operating costs. Among them, lithium battery is popular with base station batteries because of its low installation cost and long service life, and has been widely used in $5 \mathrm{G}$ base station energy storage systems ${ }^{[1][2][3]}$. Therefore, the accurate estimation of the SOC of the lithium battery has become one of the key factors to ensure the reliability and stability of the lithium battery and the entire energy storage system.

In this paper, a Dual-Polarized model is established for the lithium battery with lithium iron phosphate, and uses the least squares method with forgetting factor to carry out the online model Parameter identification; combined with the identified model parameters, the unscented Kalman filter algorithm is used to estimate the SOC of the lithium battery. Finally, the accuracy of the algorithm is verified through simulation and experiments.

\footnotetext{
* Corresponding author: lichanglei@caict.ac.cn
} 


\section{Lithium battery modeling and parameter identification}

\subsection{Dual polarization ( DP ) equivalent circuit model}

The DP model equivalent circuit of a lithium battery is shown in Figure 1.

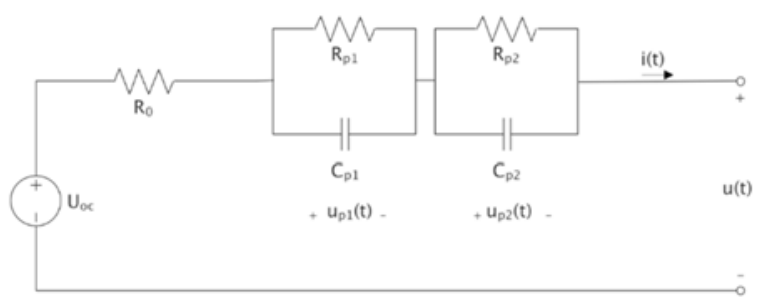

Fig. 1. Dual-polarization (DP) equivalent circuit model of lithium battery.

Selecting $u_{p 1}(t), u_{p 2}(t)$ and $S O C(t)$ as state variables, terminal current $i(t)$ of the battery as input variable, terminal voltage $u(t)$ of the battery as output variable, and discretize them to establish the discrete space state equation and observation equation of the second-order RC equivalent model, as shown in equation (1), respectively. In equations (1), $k$ is the discrete sampling point, $T_{S}$ is the time interval between two adjacent sampling points, $W_{l}(k), W_{2}(k)$ and $W_{3}(k)$ are the system white noise with a mean value of 0 and a covariance of $\mathrm{Q}(\mathrm{k}) ; \mathrm{V}(\mathrm{k})$ is a measured white noise sequence with a mean value of 0 and a covariance of $R(k)^{[4][5]}$.

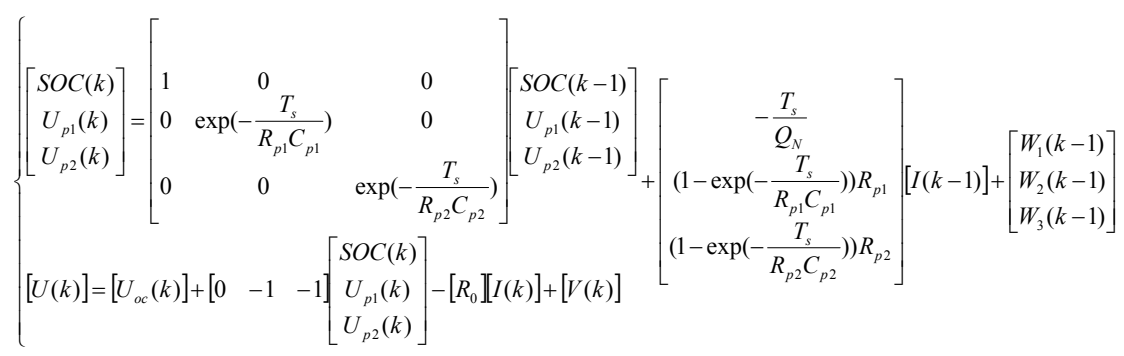

The establishment of the accurate state equation and observation equation of the secondorder lithium battery through the above has laid a good foundation for the next step of SOC estimation based on the battery model.

\subsection{Model parameter identification}

\subsubsection{Establishment of SOC-OCV relation curve}

In this paper, the battery discharge test is carried out at room temperature with $0.2 \mathrm{C}$ current, and the data of the lithium battery's standing voltage (OCV) at different SOC are obtained by the ampere-hour integral method. Then, the function relationship between SOC and OCV is obtained by curve fitting.

According to use the discharge data of SOC and $\mathrm{U}_{\mathrm{oc}}$ which is obtained from the above discharging test, it can be perform 8th-order polynomial fitting. The fitting formula obtained is shown in equation (2).

$$
\begin{gathered}
U_{o c}=-30.480 \times S O C^{8}+159.100 \times S_{O C C^{7}}-309.800 \times S O C^{6}+295.770 \times S_{O C C^{5}}-149.530 \times \\
S_{O C C^{4}}+40.624 \times S O C^{3}-6.390 \times S_{O C C}^{2}+0.928 \times S O C+3.172
\end{gathered}
$$




\subsubsection{Parameter identification based on RLS with forgetting factor}

The essence of parameter identification by recursive least square method is to calculate the error between the system output and the expectation at this time based on the result identified at the previous time, and then correct the error and newly observed data to get the corrected estimate result at the current time. The next time is followed by analogy, so as to realize the real-time update of identification parameters. Therefore, the ultimate goal is to minimize the estimation error $e_{L S}(k)$ of the system, so as to obtain the parameters that are most close to the true value ${ }^{[6][7]}$.

Based on the above analysis, the equation suitable for parameter identification based on the least square method and the error function are defined as shown in formula (3) and formula (4):

$$
\begin{gathered}
y(k)=\Phi(k) \theta(k)+e_{L S}(k) \\
E(\theta)=\sum_{i=0}^{k} \lambda^{k-i} e_{L S}{ }^{2}(k)=\sum_{i=0}^{k} \lambda^{k-i}[y(k)-\Phi(k) \theta(k)]^{2}
\end{gathered}
$$

In formula (4), in order to strengthen the influence of new observation data on the estimation results of the system, weaken the influence of old data on the estimation results of the system, and prevent data saturation, $\lambda$ is specially introduced as the forgetting factor for weighting. At the same time, in order to ensure the weighted influence of forgetting factor $\lambda$ and the stability of the whole RLS algorithm, the value range of $\lambda$ is generally $[0.95,1]^{[8]}$.

According to the foregoing analysis, in order to ensure the minimum error of the system, the minimum value of equation (4) is obtained. At the same time, according to the iterative process steps of the recursive RLS algorithm, after the system is initialized, the gain of the iterative operation is calculated first, then the covariance is calculated, then the estimation error is calculated, and finally the system estimated parameters are updated. In summary, the calculation process of the recursive least square method with forgetting factor is shown in formula (5):

$$
\left\{\begin{array}{c}
K(k)=\frac{\lambda^{-1} P(k-1) \Phi(k)}{1+\lambda^{-1} \Phi(k)^{T} P(k-1) \Phi(k)} \\
P(k)=\lambda^{-1} P(k-1)-\lambda^{-1} K(k) \Phi(k)^{T} P(k-1) \\
e_{L S}(k)=y(k)-\Phi(k) \hat{\theta}(k-1) \\
\hat{\theta}(k)=\hat{\theta}(k-1)+K(k) e_{L S}(k)
\end{array}\right.
$$

In formula (5), $K(k)$ is the gain matrix, $P(k-1)$ is the covariance matrix at the previous moment, $P(k)$ is the covariance matrix at the current moment, $\hat{\theta}(k-1)$ is the estimated value of the system parameter at the previous moment, $\hat{\theta}(k)$ is the estimated value of the system parameter at the current moment, $\Phi(k) \hat{\theta}(k-1)$ is the observed value of the system at the current moment, and $K(k) e_{L S}(k)$ is the estimated error of the system at the current moment after correction.

\subsubsection{Parameter identification results and test verification}

The hybrid pulse power characteristic (HPPC) experiment was carried out on the lithium battery. The obtained parameter identification results and parameter identification accuracy are shown in Figure 2, where (a) is the parameter identification result of $R_{0}, R_{p 1}$ and $R_{p 2}$, (b) is the parameter identification result of $\mathrm{C}_{\mathrm{p} 1}$ and $\mathrm{C}_{\mathrm{p} 2}$, and (c) is $\mathrm{R}_{0}, \mathrm{R}_{\mathrm{p} 1}, \mathrm{R}_{\mathrm{p} 2}, \mathrm{C}_{\mathrm{p} 1}$ and $\mathrm{C}_{\mathrm{p} 2}$ 
parameter identification accuracy.

Through the parameter identification results and the parameter identification accuracy curve in Figure 2, it can be seen that at the beginning of the parameter identification process, the correction factor of the identification system quickly adjusts the parameter value, so that the parameter is constantly close to the accurate value, causing large fluctuations in each parameter. After $60 \mathrm{~s}$, the error value of each parameter identification error curve decreases. With the continuous adjustment of the parameters, after $1000 \mathrm{~s}$, the fluctuation of each parameter value decreases, and the parameter identification value area is stable.

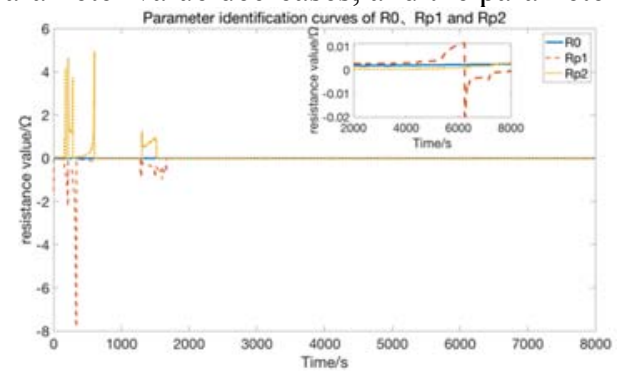

(a) Parameter identification results of $\mathrm{R}_{0}, \mathrm{R}_{\mathrm{p} 1}$

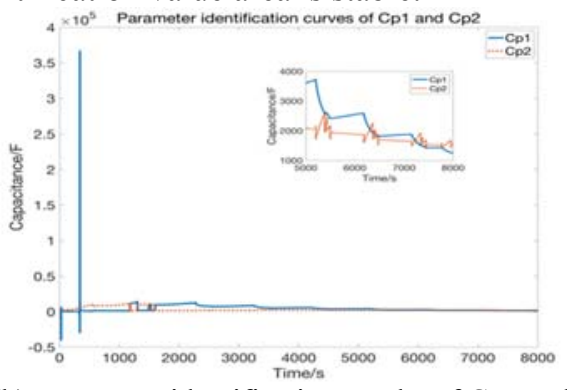

(b) Parameter identification results of $\mathrm{C}_{\mathrm{p} 1}$ and

$$
\mathrm{C}_{\mathrm{p} 2} \text { and } \mathrm{R}_{\mathrm{p} 2}
$$

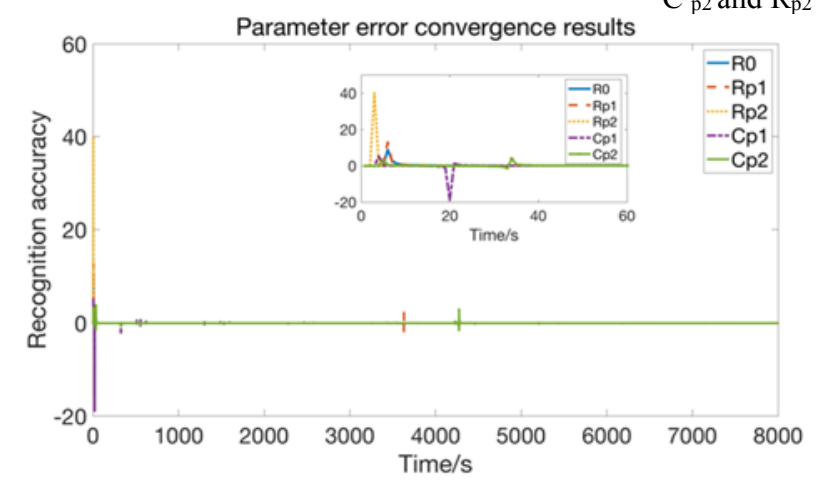

(c) Parameter identification accuracy of $\mathrm{R}_{0}, \mathrm{R}_{\mathrm{p} 1}, \mathrm{R}_{\mathrm{p} 2}, \mathrm{C}_{\mathrm{p} 1}$ and $\mathrm{C}_{\mathrm{p} 2}$

Fig. 2. Parameter identification results based on the recursive least square method with forgetting factor.

\section{SOC estimation of lithium battery}

\subsection{UKF for estimating SOC}

For the state equation and observation equation of the nonlinear discrete system shown in equation (1), defining the state quantity

$$
x_{k}=\left[\begin{array}{lll}
\operatorname{SOC}(k) & U_{p 1}(k) & U_{p 2}(k)
\end{array}\right]^{T}=\left[\begin{array}{lll}
\operatorname{SOC}_{k} & u_{p 1, k} & u_{p 2, k}
\end{array}\right]^{T}
$$

output

$$
y_{k}=[U(k)] w_{k-1}=\left[\begin{array}{l}
W_{1}(k-1) \\
W_{2}(k-1) \\
W_{3}(k-1)
\end{array}\right]
$$

observing the noise $v_{k}=[V(k)]$, and then the estimation process of untraced Kalman filter 
algorithm is as follows:

i. Initialization, including initialization of state variables and covariance:

$$
\left\{\begin{array}{c}
\hat{x}_{0}=E\left(x_{0}\right) \\
P_{0}=E\left[\left(x_{0}-\hat{x}_{0}\right)\left(x_{0}-\hat{x}_{0}\right)^{T}\right]
\end{array}\right.
$$

In Equation (6), $x_{0}$ is the initial value of the state variable, and $P_{0}$ is the initial value of the covariance.

ii. Select the appropriate Sigma sampling point and determine the weight value:

The sampling point is defined as $x_{i, k-1}$, and the weight value is $\omega_{i}$ (where $i=$ $0,1,2, \ldots, 2 n)$, then Sigma sampling points are selected as shown in formula (7) :

$$
\left\{\begin{array}{c}
x_{0, k-1}=\hat{x}_{k-1} \\
x_{i, k-1}=\hat{x}_{k-1}+\left(\sqrt{(n+\lambda) P_{k-1}}\right)_{i}, i=1,2, \ldots n \\
x_{i, k-1}=\hat{x}_{k-1}-\left(\sqrt{(n+\lambda) P_{k-1}}\right)_{i}, i=n+1, \ldots, 2 n
\end{array}\right.
$$

Through the above sampling points, the sampling weight value is calculated as shown in formula (8):

$$
\left\{\begin{array}{c}
\omega_{m}{ }^{(0)}=\frac{\lambda}{n+\lambda}, i=0 \\
\omega_{c}{ }^{(0)}=\frac{\lambda}{n+\lambda}+\left(1-\alpha^{2}+\beta\right), i=0 \\
\omega_{m}{ }^{(i)}=\omega_{c}{ }^{(i)}=\frac{1}{2(n+\lambda)}, i=1,2, \ldots, 2 n
\end{array}\right.
$$

iii. State variables and covariance time update:

$$
\left\{\begin{array}{c}
\hat{x}_{k \mid k-1}=\sum_{i=0}^{2 n} \omega_{m}{ }^{(i)} x_{i, k-1} \\
P_{k \mid k-1}=\sum_{i=0}^{2 n} \omega_{c}{ }^{(i)}\left(x_{i, k \mid k-1}-\hat{x}_{k \mid k-1}\right)\left(x_{i, k \mid k-1}-\hat{x}_{k \mid k-1}\right)^{T}+Q_{k}
\end{array}\right.
$$

iv. Mean value of the output observed by the system is updated:

$$
\left\{\begin{array}{c}
\hat{y}_{k}=\sum_{i=0}^{2 n} \omega_{m}{ }^{(i)} y_{k \mid k-1} \\
P_{y_{k} y_{k}}=\sum_{i=0}^{2 n} \omega_{c}^{(i)}\left(y_{i, k \mid k-1}-\hat{y}_{k}\right)\left(y_{i, k \mid k-1}-\hat{y}_{k}\right)^{T}+R_{k} \\
P_{x_{k} y_{k}}=\sum_{i=0}^{2 n} \omega_{c}{ }^{(i)}\left(x_{i, k \mid k-1}-\hat{x}_{k \mid k-1}\right)\left(y_{i, k \mid k-1}-\hat{y}_{k}\right)^{T}
\end{array}\right.
$$

v. Update of state variables and covariance:

$$
\left\{\begin{array}{c}
x_{k}=\hat{x}_{k \mid k-1}+P_{x_{k} y_{k}} P_{y_{k} y_{k}}{ }^{-1}\left(y_{k}-\hat{y}_{k}\right) \\
P_{k}=P_{k \mid k-1}-\left(P_{x_{k} y_{k}} P_{y_{k} y_{k}}^{-1}\right) P_{y_{k} y_{k}}\left(P_{x_{k} y_{k}} P_{y_{k} y_{k}}{ }^{-1}\right)^{T}
\end{array}\right.
$$

Through the above process from i to $\mathrm{v}$, the SOC estimation of the lithium battery of the dual polarization model can be completed ${ }^{[9][10]}$.

\subsection{SOC estimation results and experimental verification}

In order to verify the accuracy of the UKF estimation of the lithium battery SOC, before the test, the capacity of the lithium battery is calibrated to determine that the battery is fully charged with an initial capacity of $20.508 \mathrm{Ah}$, and then the HPPC pulse discharge test is carried out on the lithium battery with $0.5 \mathrm{C}(10 \mathrm{~A})$ current. Using the data obtained from the 
experiment, UKF and EKF were used to estimate the SOC of the lithium battery.

The comparison of SOC estimation curve and theoretical value obtained through UKF algorithm and EKF algorithm is shown in Figure 3(a), and the comparison of estimation error between UKF algorithm and EKF algorithm in the estimation process is shown in Figure 3(b).

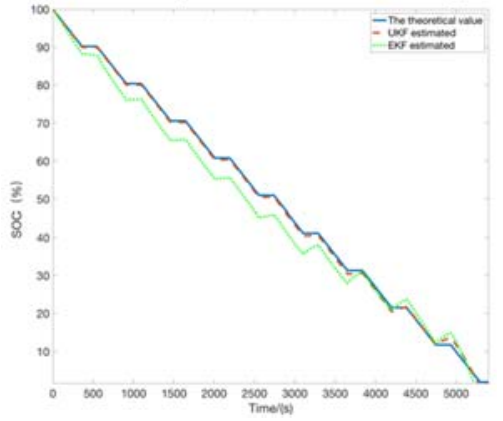

(a) Comparison between UKF and EKF SOC error curve results

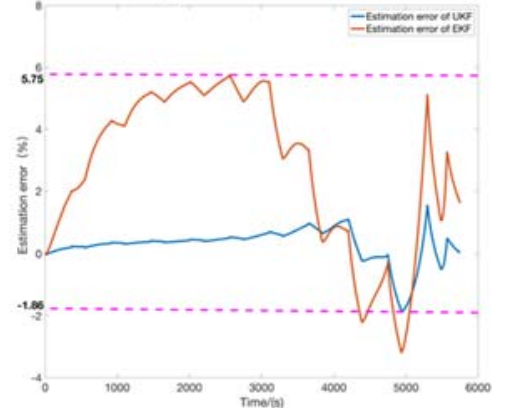

(b) Comparison between UKF and EKF estimation results and theoretical values estimated SOC

Fig. 3. UKF estimated SOC results.

It can be seen from Figure 3 (a) that the SOC curve estimated by the UKF nonlinear algorithm avoids the situation that the error caused by the first-order linearization in the traditional EKF algorithm is difficult to observe, and can track the theory stably. It can be seen from Figure 3 (b) that, compared with the EKF algorithm, the UKF algorithm has better convergence in the estimation process, and can track faster and approach the theoretical value. Compared with the maximum estimation error of the EKF which is $5.75 \%$, the maximum estimation error of the UKF algorithm is only $-1.86 \%$. The estimation results further verify the accuracy of the aforementioned lithium battery model modeling and model parameter identification.

\section{Conclusions}

In this paper, the energy storage lithium battery is modeled. RLS method with forgetting factor and UKF algorithm are used to realize the dynamic identification of model parameters and the SOC estimation of the battery respectively, and the simulation and experimental verification are carried out, and the following conclusions are reached:

1) The establishment of the DP equivalent circuit model for lithium battery is accurate and reasonable.

2) The parameter identification process of each parameter in the DP equivalent circuit model based on the lithium battery and the SOC estimation process of the lithium battery are experimentally studied.

3) Through the analysis of the simulation and test results, it is believed that the SOC of lithium battery can be accurately estimated by using the DP equivalent circuit model parameters and UKF SOC estimation, and the estimation accuracy can meet the real-time use requirements of $5 \mathrm{G}$ base station standby power.

\section{References}

1. Li Jianlin, Tan Yuliang, Wang Nan, Wang Han, Wang Li . Analysis of typical application scenarios of energy storage technology under new infrastructure [J/OL]. Thermal Power Generation, 2020, 6:3-4.

2. Liu Dahe, Han Xiaojuan, Li Jianlin . Economic analysis of cascade battery energy 
storage based on photovoltaic power station scenario [J]. Electric Power Engineering Technology, 2017, 36(6): 27.

3. Lin Quan. The fundamental direction of low-carbon economic building materials industry structure adjustment [J]. China Building Materials, 2009(9): 24-29.

4. Wang Ming, Li Jianjun, Wu defend and so on . Research progress in lithium battery model [J]. Power Technology, 2011 (7): 862-86.

5. Han Zongqi , Liuji Liang, Bo Zhu et . Ferric phosphate lithium battery at typical temperatures PNGV Model [J]. Yanshan University, 2012,36 (3): 248-253.

6. Li Kuangcheng, Yang Yali, Chen Tao, et al . Online estimation of battery state of charge with least square method [J]. Power Supply Technology , 2010, 34(10): 10561059.

7. Shi Xianliang, Wu Chengfu . Based on MATLAB Parameter Identification and simulation least square method [J]. Microprocessor, 2005,26 (6): 44-46.

8. Chen Han, Liu Huijin, Li Dalu, et al . Variable forgetting factor recursive least square method to measure time-varying parameters [J]. High Voltage Technology , 2008, 34(7): 1474-1477.

9. Guo Xiangwei . Research on State of Charge Estimation and Equalization Technology of Electric Vehicle Battery [D]. South China University of Technology , 2016: 38-40.

10. Zhu Mengjie . Estimation of the state of charge of lithium batteries for electric vehicles [D]. Anhui University of Science and Technology , 2019: 43-46. 